\title{
MicroRNA-150 upregulation reduces osteosarcoma cell invasion and metastasis by downregulating Ezrin
}

\author{
CE ZHAN*, CHENG LI*, HAO ZHANG, HAO TANG, FANG JI, \\ SU-CHI QIAO, WEI-DONG XU and ZHI-WEI WANG
}

Department of Orthopedics, Changhai Hospital, The Second Military Medical University, Shanghai 200433, P.R. China

Received October 25, 2014; Accepted August 5, 2015

DOI: $10.3892 / \mathrm{ol} .2016 .5046$

\begin{abstract}
The present study aimed to investigate the effect of microRNA-150 (miRNA/miR-150) in osteosarcoma (OS) cell invasion and metastasis by the regulation of Ezrin. To compare the differences in the expression of miR-150 and Ezrin, cell models of OS metastasis were established by exogenous transfection of miR-150 on the basis of different expression levels of miR-150. Reverse transcription-quantitative polymerase chain reaction (RT-qPCR) was used to estimate these expression levels. Ezrin expression was detected by western blot assay. Methylthiazolyldiphenyl-tetrazolium bromide assay was performed to determine cells proliferation. Cell invasion and migration were measured in vitro by Transwell migration assays. Detection of apoptosis adopted flow cytometry. The results of RT-qPCR showed that the miR-150 expression in OS F5M2 cells was significantly increased following exogenous transfection of miR-150 mimics, and the expression of miR-150 was positively correlated with the concentration of the miR-150 mimics. Western blot assay indicated that the Ezrin expression in the F5M2 cells was decreased with the exogenous overexpression of miR-150. Additionally, Transwell assays revealed that the overexpression of miR-150 significantly suppressed the invasion and metastasis ability of the F5M2 cells. miR-150 upregulation may reduce OS cell invasion and metastasis by downregulating the expression of Ezrin.
\end{abstract}

\section{Introduction}

Osteosarcoma (OS), known as the most common primary malignant tumor arising from bone, is derived from mesenchymal cells, which generate osteoid and immature bone (1-3). It has been demonstrated that OS has a significant

Correspondence to: Dr Zhi-Wei Wang, Department of Orthopedics, Changhai Hospital, The Second Military Medical University, 168 Changhai Road, Yangpu, Shanghai 200433, P.R. China

E-mail: wangzhiwei1025@126.com

${ }^{*}$ Contributed equally

Key words: osteosarcoma, microRNA-150, invasion and metastasis age distribution; the first peak appears during adolescence (10-14 years old), and the second peak occurs in elderly adults (>65 years) (1). Although advanced treatments, such as surgery, chemotherapy and radiotherapy, are used, the 5-year survival rate of OS patients is $60-70 \%$ (4). It has been reported that the morbidity of males diagnosed with OS is 1.4 times higher than that of females (5). Furthermore, OS mainly occurs in the long bones, particularly in the metaphyses, proximal tibia, proximal humerus and distal femur, while $\sim 10 \%$ of OS occurs in the axial skeleton, most frequently in the pelvis $(5,6)$. Meanwhile, $\sim 30 \%$ of OS patients have the potential for pulmonary metastatic disease, and the 5-year survival rates are considered to be $<20 \%$ (7). It has been shown that the pathogenesis of OS contributes to the interactions between genetic and environmental risk factors, including smoking, air population and ionizing radiation $(4,8-10)$. Numerous studies have focused on the association of microRNAs (miRNAs/miRs) and the development of OS and its underlying mechanisms $(1,10)$.

miRNAs are a class of novel small non-coding RNA molecules that can regulate the gene expression of target mRNA in animals and plants (11-13). Previous studies have suggested that miRNAs exhibit significant functions in certain physiological processes, including cell proliferation, differentiation, apoptosis, angiogenesis, stress resistance and the self-renewal of stem cells $(14,15)$. In addition, miRNAs may function as tumor suppressors and oncogenes in specific cases, and certain miRNAs are also viewed as candidate targets for intervention therapy or molecular markers for the early diagnosis and prognosis of tumors (16-18). Recently, studies have demonstrated that various miRNAs, including miR-125b, miR-143, miR-20a, miR-199a-3p, miR-34a and miR-150, may be closely associated with tumor metastasis and invasion in OS $(19,20)$. Among them, miR-150, which is abundant in lymphocytes, acts as a sensitive marker that indicates bone marrow damage and lymphocyte depletion $(21,22)$. It is well documented that miR-150 plays an essential role as an oncogene in different tumors, including gastric cancer, OS, lung cancer and hepatoblastoma $(23,24)$. Ezrin, known as a member of the Ezrin-Radixin-Moesin protein family, is a cytoskeleton linkage protein that actively participates in the regulation of growth and metastasis of cancer cells (25). Ezrin is also defined as a predominant component of the surface structure of cells, being involved in adhesion to the extracellular matrix, cell-to-cell communication and the signaling 
pathway of receptor tyrosine kinase (26). Due to the function of membrane-cytoskeleton linking, Ezrin has been found to be essential for the metastasis of OS in a rat model, while the high expression of Ezrin in dogs with OS has also been correlated with the early progress of metastasis (27). The present study aimed to investigate the effect of miRNA-150 on the proliferation and metastasis of the human OS F5M2 cell line, in order to provide a basis for the treatment of OS.

\section{Materials and methods}

Cells culture and grouping design. The human OS F5M2 cell line was acquired from the American Type Culture Collection (Manassas, VA, USA) and cultured in RPMI-1640 medium that contained $10 \%$ fetal bovine serum (FBS; Gibco, Life Technologies, Carlsbad, CA, USA), 100 U/ml penicillin $\mathrm{G}$ and $100 \mathrm{U} / \mathrm{ml}$ streptomycin (Invitrogen, Life Technologies, Carlsbad, CA, USA) at $37^{\circ} \mathrm{C}$ with $5 \% \mathrm{CO}_{2}$. The optimal F5M2 subline during logarithmic growth phase was harvested with $0.05 \%$ trypsin (containing $0.02 \%$ EDTA). The harvested F5M2 subline samples were assigned to five groups: i) Control group (untreated F5M2 cell subline); ii) miR-150 low-dose group (F5M2 cells transfected with $20 \mathrm{nM}$ miR-150 mimics); iii) miR-150 high-dose group (F5M2 cells transfected with $50 \mathrm{nM}$ miR-150 mimics); iv) negative control (NC) group (F5M2 cells transfected with $20 \mathrm{nM}$ miR-150 NC, acting as the control of the miR-150 mimics group and the miR-150 inhibitors group); v) inhibitor group (F5M2 cells transfected with $20 \mathrm{nM}$ miR-150 inhibitor). miR-150 mimic, miR-150-negative control and miR-150 inhibitor were provided by Guangzhou RiboBio Co., Ltd. (Guangzhou, China).

The cells were cultured in $50 \mathrm{ml}$ complete culture medium, and grown to $30-50 \%$ density. Lipofectamine ${ }^{\circledR} 2000$ (Invitrogen, Life Technologies) and miR-150 mimic/miR-150 inhibitor/miR-150 negative control were prepared in a sterile Eppendorf tube (Eppendorf microfuge, Brinkmann Instruments, Inc., Westbury, NY, USA). The diluted miR-150 mimic/miR-150 inhibitor/miR-150 negative control and Lipofectamine 2000 were mixed gently to a total volume of $100 \mu \mathrm{l}$, and incubated at room temperature for $20 \mathrm{~min}$. A total of $100 \mu \mathrm{l}$ of transfection solution was added to each cell with gentle agitation. Cells were subsequently incubated at $37^{\circ} \mathrm{C}$ in an atmosphere of $5 \% \mathrm{CO}_{2}$, followed by removal of transfection solution and replacement with complete medium 6-8 h later. Following 48 to $72 \mathrm{~h}$ of culture, the cells were harvested and the total protein was extracted. The experiment was repeated 3 times for each group.

The study was approved by the ethics committee of the Second Military Medical University (Shanghai, China).

Proliferation assay. Transfected F5M2 cells of each group were seeded in seven 96 -well plates $\left(1 \times 10^{4}\right.$ cells per well) using methylthiazolyldiphenyl-tetrazolium bromide (MTT) assay (Sigma-Aldrich, St. Louis, MO, USA). The absorbance (OD value) was determined at $490 \mathrm{~nm}$ (one plate per day) on a microplate spectrophotometer (SpectraMax, Molecular Devices, Sunnyvale, CA, USA). After seven days, the average absorbance was calculated. Cell growth curves were obtained for the comparison of growth conditions among groups.
Cell migration in vitro. Transwell inserts (24-well insert; pore size, $8 \mu \mathrm{m}$ ) were applied to investigate the effect of miR-150 on the migration and invasion of the F5M2 cells. Transwell inserts were coated with Matrigel (50 $\mu \mathrm{l}$ per filter) (BD Biosciences, Franklin Lakes, NJ, USA) based on the manufacturer's instructions. Following transfection, the F5M2 cells were transferred onto the Matrigel-coated upper inserts (at a density of $1 \times 10^{5}$ per Transwell), which contained serum-free Dulbecco's modified Eagle's medium (DMEM) and then incubated at $37^{\circ} \mathrm{C}$ for $48 \mathrm{~h}$. DMEM containing $10 \%$ FBS was added to the lower inserts and further incubation was performed for $24 \mathrm{~h}$. The cells which migrated to the lower inserts were fixed with $90 \%$ ethanol and stained with crystal violet, and the cells that remained on the top of the filters were scrubbed off. Six random visual fields of each insert were counted subsequent to being observed under a microscope. Migration assay was performed in the same manner as the aforementioned procedures but without the Matrigel coating. The cells that penetrated the upper side of the membrane were fixed with ethanol and stained. Finally, the cells were counted under a microscope (Eclipse E600; Nikon Inc., Melville, NY, USA).

Apoptosis test. Apoptotic cells were analyzed by double staining with Annexin V-FITC/PI Apoptosis Assay (BD Biosciences Pharmingen, San Diego, CA, USA). Following transfection, adherent cells were trypsinized, centrifuged (400 x g) and washed three times in phosphate-buffered saline. The cells were resuspended with $300 \mu$ l staining solution containing fluorescein isothiocyanate (FITC)-conjugated Annexin $\mathrm{V}$ antibody and propidium iodide (PI). Incubation was performed on ice for $15 \mathrm{~min}$ and the cells were analyzed using flow cytometry (BD Biosciences). The number of apoptotic cells (Annexin ${ }^{+} \mathrm{PI}^{-}$) was recorded by applying CellQuest Software (BD Biosciences Pharmingen).

Reverse transcription-quantitative polymerase chain reaction $(R T-q P C R)$. Total RNA was isolated using TRIzol reagent (Invitrogen, Life Technologies) and then reverse transcription was performed. Designed PCR primers for miR-150 were as follows: Forward, 5'-TGTCCTGCGGGAGTGGTA-3' and reverse, 5'-TGATGCTGGTGGGTCTGC-3'. The length of the PCR product was $387 \mathrm{bp}$. Primers were synthesized by Invitrogen (Shanghai, China). PCR conditions were predenaturation at $95^{\circ} \mathrm{C}$ for $5 \mathrm{~min}$, denaturation at $94^{\circ} \mathrm{C}$ for $50 \mathrm{sec}$, annealing at $58^{\circ} \mathrm{C}$ for $50 \mathrm{sec}$ and elongation at $72^{\circ} \mathrm{C}$ for $1 \mathrm{~min}$, for a total of 30 cycles. PCR products were separated by electrophoresis on $2 \%$ agarose gels and then the results were analyzed.

Western blotting. Total proteins were extracted and detected by western blot analysis. For further study, the protein was electrophoresed by $12 \%$ SDS-PAGE, then transferred onto polyvinylidene difluoride (PVDF) membranes (EMD Millipore, Billerica, MA, USA). Subsequent to being blocked with $10 \%$ skimmed milk for $1 \mathrm{~h}$, the membranes were washed with TBS. The goat polyclonal antibody against ezrin (C-15; dilution, 1:200; Santa Cruz Biotechnology Inc., Dallas, TX, USA), was added at a 1:1,000 dilution, using U6 as the internal control; horseradish peroxidase (HRP)-labeled immunoglobulin G (Jackson ImmunoResearch, West Grove, PA, USA) was supplemented as the secondary antibody at a 1:1,000 dilution. 
Table I. Expression of miR-150 in each group.

\begin{tabular}{lcccc}
\hline Group & mean \pm SD & MD & 95\% CI & \\
\hline Control & $19.67 \pm 2.16$ & & & \\
Negative control & $17.33 \pm 4.50$ & -2.34 & -5.66 to 10.34 & 0.462 \\
miR-150 low-dose & $66.00 \pm 10.08$ & 46.33 & -62.85 to -29.81 & 0.002 \\
miR-150 high-dose & $75.17 \pm 8.23$ & 55.50 & -69.14 to -41.86 & $<0.001$ \\
Inhibitor & $7.83 \pm 2.71$ & -11.84 & 6.29 to 17.39 & 0.004 \\
\hline
\end{tabular}

Comparisons are in contrast with the control group. miR-150, microRNA-150; SD, standard deviation; MD, mean difference; CI, confidence interval.

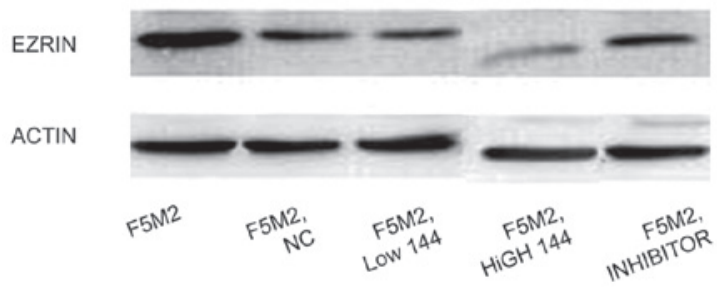

Figure 1. Western blotting for Ezrin protein expression.

Subsequently, chemiluminescence immunoblotting detection reagents were applied to the PVDF membranes. X-ray was utilized for exposure and visualization, and then the expression level of Ezrin was analyzed by the intensity of electrophoresis.

Statistical analysis. Statistical analyses were conducted using SPSS 18.0 software (SPSS, Inc., Chicago, IL, USA). Data were expressed as the mean \pm standard deviation. Measurement data were by compared by applying a t-test. $\mathrm{P}<0.05$ was considered to indicate a statistically significant difference.

\section{Results}

Expression of miR-150 and Ezrin protein. miR-150 expression in each group was detected by RT-qPCR and the $2^{-\Delta \Delta C T}$ method. Higher miR-150 expression levels were detected in the miR-150 low-dose and high-dose groups compared with the control group at $48 \mathrm{~h}$ post-transfection (both $\mathrm{P}<0.05$ ). When compared with the control group, the expression of miR-150 was evidently decreased (both $\mathrm{P}<0.05$ ), while there was no statistically significant difference between the control and the NC groups $(\mathrm{P}>0.05)$ (Table I). The level of Ezrin expression in each group was detected by western blot analysis. It was observed that Ezrin expression levels in the low-dose and high-dose groups were notably lower than that in the control group, and the expression of Ezrin in the high-dose group was markedly reduced compared with the low-dose group (Fig. 1).

Migration ability of F5M2 cells and transfected F5M2 cells. A paired samples t-test was applied to compare the migration ability through the poly-carbonate membrane in the Transwell experiment. Subsequent to being transfected with miR-150, the migration ability of the miR-150 low-dose and high-dose groups decreased in contrast to the F5M2 control cells without
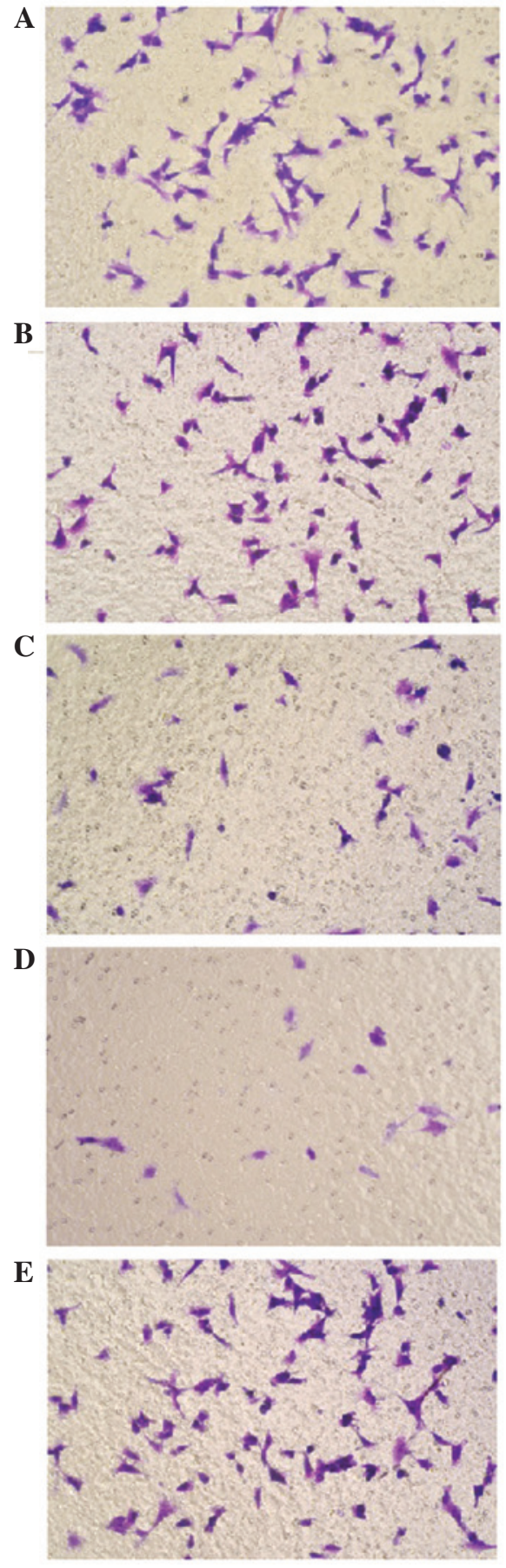

Figure 2. Transwell experiment in each group. (A) Control; (B) negative control; (C) miR-150 low-dose; (D) miR-150 high-dose; and (E) inhibitor. 


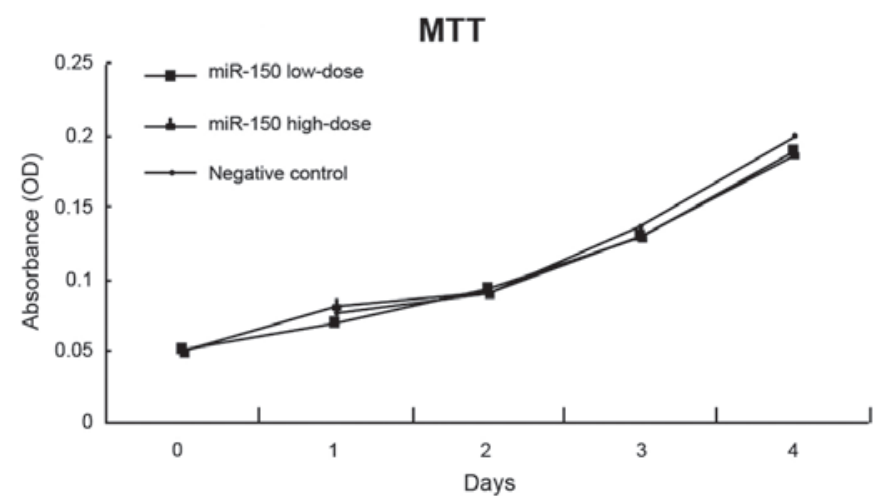

Figure 3. Proliferation of F5M2 cells, as determined by MTT assay. OD, optical density. MTT, methylthiazolyldiphenyl-tetrazolium bromide; miR, microRNA; OD, optical density.

A

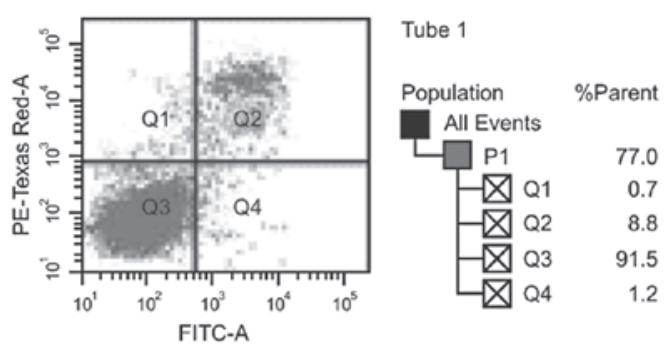

B

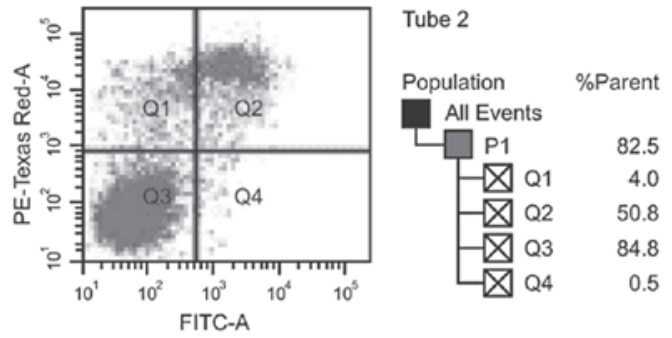

C

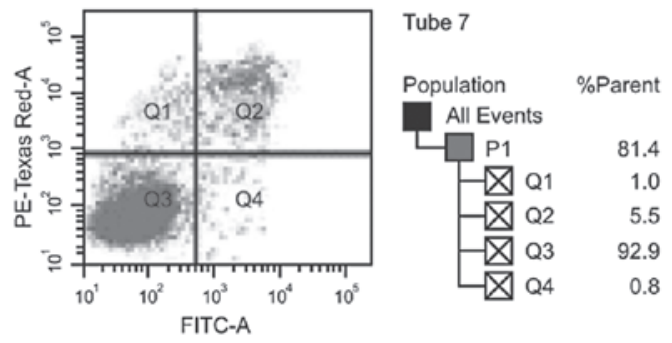

Figure 4. Apoptosis of F5M2 cells, as determined by flow cytometry. (A) miR-150 low-dose. (B) miR-150 high-dose. (C) negative control. FITC, fluorescein isothiocyanate.

transfection. Compared with the control group, no significant effect on migration ability was found in the NC and inhibitor groups (both $\mathrm{P}>0.05$ ) (Fig. 2).

Proliferation and apoptosis of F5M2 cells. The absorbance of untreated F5M2 cells, F5M2 cells transfected with high-dose miR-150 mimics, F5M2 cells transfected with low-dose miR-150 mimics, F5M2 cells transfected with inhibitors and F5M2 cells in the NC group were continuously detected for seven days, and then growth curves were drawn (Fig. 3).
According to the MTT assay, the cell growth curves of the F5M2 cells in the different groups were close to each other, indicating no marked differences between the proliferation of the groups $(\mathrm{P}>0.05)$. The cell growth curves of the F5M2 cells in the high-dose and control groups also showed no significant differences $(\mathrm{P}>0.05)$. Fig. 4 shows that the apoptotic capacity of the high-dose and low-dose miR-150-transfected F5M2 cells did not differ from that of the control F5M2 cells upon flow cytometry $(\mathrm{P}>0.05)$. Additionally, no marked statistical difference in the total apoptosis rate was observed between the $\mathrm{NC}$ and control groups $(\mathrm{P}>0.05)$.

\section{Discussion}

The results of the present study showed that the expression of miR-150 in the OS cells significantly improved after the exogenous transfection of miR-150 mimics. Watanabe et al reported that miR-150 can suppress malignant lymphoma by activating the phosphoinositide 3-kinase-Akt pathway (28). Yokobori et al observed that regulation of miR-150 expression in esophageal squamous cell carcinoma could inhibit malignant potential, such as lymph node metastasis and invasion, by controlling the activity of zinc finger E-box binding homeobox 1 (29). Thus, analysis of previous studies concluded that the expression of miR-150 in highly metastatic OS is lower than that in less metastatic OS. It was also indicated that the expression of miR-150 in F5M2 cells may be improved through the transfection of miR-150 mimics (30). Similarly, the present study suggested that the expression of miR-150 shows a positive association with the transfection concentration of miR-150 mimics.

Another pivotal result from the present study was that the exogenous overexpression of miR-150 may lead to a significant decrease in the invasion and metastasis ability of F5M2 cells, indicating a negative dose-dependent association. In addition, miR-150 had no significant effect on the ability for proliferation and apoptosis in the F5M2 cells. One potential explanation for this may be that the overexpression of miR-150 could contribute significantly to the reduction in the migration and invasion ability of OS F5M2 cells in vitro; the relatively higher concentration of exogenous miR-150 appears to be correlated with the inhibitory effect on the migration and invasion of tumor cells $(25,31)$. Studies have demonstrated that the metastatic ability of OS is inversely associated with the expression levels of miR-150. Additionally, the expression level of miR-150 is inversely associated with the expression of Ezrin, as observed in the present study, suggesting that miR-150 may inhibit the expression of Ezrin to a certain extent $(32,33)$.

Ezrin has been suggested to play a role in the physical and functional connections between the actin cytoskeleton and the cell membrane (34). Moreover, Ezrin also has effects on maintaining cell shape and cell polarity, participating in membrane-interacting pathways, and the migration, signaling, growth regulation and differentiation of cells; due to the unique functions of Ezrin, the protein has been considered to be involved in the metastatic capacity of cancer (25). According to the result of the present study, it was concluded that the level of Ezrin in cells may not only be the marker that distinguishes tumors from non-tumors, but also that it 
may predict the metastatic potential of tumor cells, which is of significance for clinical guidance in the estimation of OS metastasis and its prognosis. Moreover, the level of Ezrin expression is positively correlated with the metastatic ability of OS, suggesting that a decrease in Ezrin expression is a positive predictive factor of the overall survival rate of patients with OS (27). Therefore, the expression of Ezrin can be considered as a novel area research for the treatment of OS metastasis (35). We also postulate that the expression of Ezrin may be adversely associated with miR-150 expression. Accordingly, the exogenous overexpression of miR-150 may decrease the metastasis of OS through downregulating the expression of Ezrin (30). Furthermore, with an increase in exogenous miR-150, the inhibition of Ezrin expression secreted by F5M2 cells becomes more apparent (36). Thus, there may be a dependent association between the inhibition of Ezrin expression and the dosage of exogenous miR-150 in F5M2 cells.

However, certain limitations existed in the present study that may merit a further investigation. Firstly and importantly, the sample size may have been too small to adequately address the role of exogenous miR-126 in OS via targeting Ezrin expression. Moreover, the retrospective study design will have affected the grouping of the cases and controls. With respect to those limitations, larger multi-center randomized controlled trials are necessary for the verification of these findings.

Collectively, miR-150 was negatively correlated with the invasion and metastatic ability of the OS cells, while a positive correlation was found with Ezrin expression. The elevated exogenous expression of miR-150 could inhibit the invasion and metastatic ability of F5M2 cells via negatively regulating Ezrin and have no significant effect on proliferation and apoptosis, in turn providing a novel molecular target for the therapeutic intervention in OS.

\section{Acknowledgements}

The authors would like to acknowledge the reviewers of this study for providing useful comments on the original manuscript.

\section{References}

1. Ottaviani G and Jaffe N: The epidemiology of osteosarcoma. Cancer Treat Res 152: 3-13, 2009.

2. Tang N, Song WX, Luo J, Haydon RC and He TC: Osteosarcoma development and stem cell differentiation. Clin Orthop Relat Res 466: 2114-2130, 2008.

3. Ta HT, Dass CR, Choong PF and Dunstan DE: Osteosarcoma treatment: State of the art. Cancer Metastasis Rev 28: 247-263, 2009.

4. Broadhead ML, Clark JC, Myers DE, Dass CR and Choong PF: The molecular pathogenesis of osteosarcoma: A review. Sarcoma 2011: 959248, 2011.

5. Ritter J and Bielack SS: Osteosarcoma. Ann Oncol 21 (Suppl 7): vii320-vii325, 2010.

6. Corradi D, Wenger DE, Bertoni F, Bacchini P, Bosio S, Goldoni M, Unni KK, Sim FH and Inwards CY: Multicentric osteosarcoma: Clinicopathologic and radiographic study of 56 cases. Am J Clin Pathol 136: 799-807, 2011.

7. Lussier DM, O'Neill L, Nieves LM, McAfee MS, Holechek SA, Collins AW, Dickman P, Jacobsen J, Hingorani P and Blattman JN: Enhanced T-cell immunity to osteosarcoma through antibody blockade of PD-1/PD-L1 interactions. J Immunother 38: 96-106, 2015.

8. Gorlick R: Current concepts on the molecular biology of osteosarcoma. Cancer Treat Res 152: 467-478, 2009.
9. Mirabello L, Pfeiffer R, Murphy G, Daw NC, Patiño-Garcia A Troisi RJ, Hoover RN, Douglass C, Schüz J, Craft AW and Savage SA: Height at diagnosis and birth-weight as risk factors for osteosarcoma. Cancer Causes Control 22: 899-908, 2011.

10. Jones KB, Salah Z, Del Mare S, Galasso M, Gaudio E, Nuovo GJ, Lovat F, LeBlanc K, Palatini J and Randall RL: miRNA signatures associate with pathogenesis and progression of osteosarcoma. Cancer Res 72: 1865-1877, 2012.

11. O'Connell RM, Rao DS and Baltimore D: microRNA regulation of inflammatory responses. Annu Rev Immunol 30: 295-312, 2012.

12. Reid G, Kirschner MB and van Zandwijk N: Circulating microRNAs: Association with disease and potential use as biomarkers. Crit Rev Oncol Hematol 80: 193-208, 2011.

13. Wu Q, Jin H, Yang Z, Luo G, Lu Y, Li K, Ren G, Su T, Pan Y, Feng B, et al: MiR-150 promotes gastric cancer proliferation by negatively regulating the pro-apoptotic gene EGR2. Biochem Biophys Res Commun 392: 340-345, 2010.

14. Baraniskin A, Kuhnhenn J, Schlegel U, Chan A, Deckert M, Gold R, Maghnouj A, Zöllner H, Reinacher-Schick A, Schmiegel W, et al: Identification of microRNAs in the cerebrospinal fluid as marker for primary diffuse large B-cell lymphoma of the central nervous system. Blood 117: 3140-3146, 2011.

15. Turchinovich A, Weiz L, Langheinz A and Burwinkel B: Characterization of extracellular circulating microRNA. Nucleic Acids Res 39: 7223-7233, 2011.

16. McDermott AM, Heneghan HM, Miller N and Kerin MJ: The therapeutic potential of microRNAs: Disease modulators and drug targets. Pharm Res 28: 3016-3029, 2011.

17. Mendell JT and Olson EN: MicroRNAs in stress signaling and human disease. Cell 148: 1172-1187, 2012.

18. Olson EN: MicroRNAs as therapeutic targets and biomarkers of cardiovascular disease. Sci Trans1 Med 6: 239ps3, 2014.

19. Zhou X, Wei M and Wang W: MicroRNA-340 suppresses osteosarcoma tumor growth and metastasis by directly targeting ROCK1. Biochem Biophys Res Commun 437: 653-658, 2013.

20. Bao YP, Yi Y, Peng LL, Fang J, Liu KB, Li WZ and Luo HS: Roles of microRNA-206 in osteosarcoma pathogenesis and progression. Asian Pac J Cancer Prev 14: 3751-3755, 2013.

21. Jacob NK, Cooley JV, Yee TN, Jacob J, Alder H, Wickramasinghe $\mathrm{P}$, Maclean $\mathrm{KH}$ and Chakravarti A: Identification of sensitive serum microRNA biomarkers for radiation biodosimetry. PLoS One 8: e57603, 2013.

22. Zhang Y, Liu D, Chen X, Li J, Li L, Bian Z, Sun F, Lu J, Yin Y, Cai X, et al: Secreted monocytic miR-150 enhances targeted endothelial cell migration. Mol Cell 39: 133-144, 2010.

23. Bezman NA, Chakraborty T, Bender T and Lanier LL: miR-150 regulates the development of NK and iNKT cells. J Exp Med 208: 2717-2731, 2011.

24. Zhang N, Wei X and Xu L: miR-150 promotes the proliferation of lung cancer cells by targeting P53. FEBS Lett 587: 2346-2351, 2013.

25. Park HR, Jung WW, Bacchini P, Bertoni F, Kim YW and Park YK: Ezrin in osteosarcoma: comparison between conventional high-grade and central low-grade osteosarcoma. Pathol Res Pract 202: 509-515, 2006.

26. Hunter KW: Ezrin, a key component in tumor metastasis. Trends Mol Med 10: 201-204, 2004.

27. Khanna C, Wan X, Bose S, Cassaday R, Olomu O, Mendoza A, Yeung C, Gorlick R, Hewitt SM and Helman LJ: The membrane-cytoskeleton linker ezrin is necessary for osteosarcoma metastasis. Nat Med 10: 182-186, 2004.

28. Watanabe A, Tagawa H, Yamashita J, Teshima K, Nara M, Iwamoto K, Kume M, Kameoka Y, Takahashi N, Nakagawa T, et al: The role of microRNA-150 as a tumor suppressor in malignant lymphoma. Leukemia 25: 1324-1334, 2011.

29. Yokobori T, Suzuki S, Tanaka N, Inose T, Sohda M, Sano A, Sakai M, Nakajima M, Miyazaki T, Kato H and Kuwano H: MiR-150 is associated with poor prognosis in esophageal squamous cell carcinoma via targeting the EMT inducer ZEB1. Cancer Sci 104: 48-54, 2013.

30. Miao J, Wu S, Peng Z, Tania M and Zhang C: MicroRNAs in osteosarcoma: Diagnostic and therapeutic aspects. Tumour Biol 34: 2093-2098, 2013.

31. Cai H, Lin L, Cai H, Tang M and Wang Z: Prognostic evaluation of microRNA-210 expression in pediatric osteosarcoma. Med Oncol 30: 499, 2013.

32. Zhao H, Guo M, Zhao G, Ma Q, Ma B, Qiu X and Fan Q: miR-183 inhibits the metastasis of osteosarcoma via downregulation of the expression of Ezrin in F5M2 cells. Int J Mol Med 30: 1013-1020, 2012. 
33. Zhu J, Feng Y, Ke Z, Yang Z, Zhou J, Huang X and Wang L: Down-regulation of miR-183 promotes migration and invasion of osteosarcoma by targeting Ezrin. Am J Pathol 180: 2440-2451, 2012.

34. Neisch AL and Fehon RG: Ezrin, radixin and moesin: Key regulators of membrane-cortex interactions and signaling. Curr Opin Cell Biol 23: 377-382, 2011.
35. Clark JC, Dass CR and Choong PF: A review of clinical and molecular prognostic factors in osteosarcoma. J Cancer Res Clin Oncol 134: 281-297, 2008.

36. Kafchinski LA and Jones KB: MicroRNAs in osteosarcomagenesis. Adv Exp Med Biol 804: 119-127, 2014. 